\section{Abstract}

\title{
Optimising configuration of a hyperspectral imager for on-line field
}

\section{measurement of wheat canopy}

\author{
Rebecca L Whetton ${ }^{\mathrm{a}}$, Toby W Waine ${ }^{\mathrm{a}}$, and Abdul M Mouazen ${ }^{\mathrm{a}, \mathrm{b} *}$ \\ ${ }^{a}$ Cranfield Soil and AgriFood Institute, Cranfield University, Bedfordshire MK43 OAL, UK. \\ ${ }^{\mathrm{b}}$ Department of Soil Management, Ghent University, Coupure 653, 9000 Gent, Belgium. \\ E-mail of corresponding author: Abdul.Mouazen@UGent.be
}

There is a lack of information on optimal measurement configuration of hyperspectral imagers for on-line measurement of a wheat canopy, this paper aims at identifying this configuration using a passive sensor $(400-750 \mathrm{~nm})$. The individual and interaction effects of camera height and angle, sensor integration time and light source distance and height on the spectra's signal-to-noise ratio (SNR) were evaluated under laboratory scanning conditions, from which an optimal configuration was defined and tested under on-line field measurement conditions. The influences of soil total nitrogen (TN) and moisture content (MC) measured with an on-line visible and near infrared (vis-NIR) spectroscopy sensor on SNR were also studied. Analysis of variance and principal component analysis (PCA) were applied to understand the effects of the laboratory considered factors and to identify the most influencing components on SNR.

Results showed that integration time and camera height and angle are highly influential factors affecting SNR. Among integration times of 10, 20 and $50 \mathrm{~ms}$, the highest SNR was obtained with $1.2 \mathrm{~m}, 1.2 \mathrm{~m}$ and $10^{\circ}$ values of light height, light distance and camera angle, respectively. The optimum integration time for on-line field measurement was $50 \mathrm{~ms}$, 
obtained at an optimal camera height of $0.3 \mathrm{~m}$. On-line measured soil TN and MC were found

to have significant effects on the SNR with Kappa values of 0.56 and 0.75 , respectively. In conclusion, an optimal configuration for a tractor mounted hyperspectral imager was established for the best quality of on-line spectra collected for wheat canopy.

\section{Keywords}

Hyperspectral imager, signal-to-noise ratio, wheat canopy, principal component analysis, soil properties.

\section{Introduction}

Advanced methods for early disease detection in crops is vital for improving the efficacy of treatment, reducing infection and minimising the losses to yield and quality. Traditionally, disease detection is carried out manually, which is costly, time consuming and requires special expertise (Schmale \& Bergstrom, 2003; Bock et al., 2010). Developments in agricultural technology have led to demands for a non-destructive, automated approach for crop disease detection that should be ideally rapid, disease specific, and sensitive to early symptoms (López et al., 2003). Optical sensing methods are non-destructive, allowing repeated data acquisition throughout the growing season without inhibiting crop growth. Spectroscopy and imaging techniques have been used in disease and stress monitoring (Hahn, 2009). However, their in-situ application although established in other industries (e.g. health services, pharmacology and food safety) is still rather limited. Both Lenk et al. (2007) and Sankaran et al. (2010) focused on implementing the technology in the field, as a mobile (on-line) 
application for mapping crop disease. Yuan et al. (2016) have used high spatial satellite imagery in the detection of powdery mildew. Remote spectral sensing for identification of weeds in wheat fields has been tested by means of ground collected data (Gómez-Casero et al., 2009). Herrmann et al. (2013) have applied proximal hyperspectral imagery in the field for weed detection (e.g., both broadleaf and grass weeds), reporting $85 \%$ accuracy. Okamoto \& Lee (2009) collected in situ hyperspectral images for the detection of green citrus fruits, reporting promising results for identification of citrus fruits from background objects. In contrast, non-mobile (off-line) and laboratory methods for disease classification and plant growing conditions have been studied and demonstrated (Roggo et al., 2003; Wu et al., 2008). Hahn (2009) claims that spectroscopic and imaging techniques could be integrated with agricultural vehicles, providing non-invasive and reliable systems for the monitoring and mapping of crop diseases, with further potential for early disease detection. Moshou et al. (2005) have shown that hyperspectral imaging for the recognition of in-situ disease can provide identification with a high degree of accuracy. Depending on the method of analysis and data fusion, an error between 1 - 16.5\% was reported.

Spectral reflectance in vegetation canopies is dependent on several factors including the illumination angle, the canopy architecture and the radiative properties of the plants. The reflectance of crop canopies is non-lambertian scattering, varying with the sun position, view positions and meteorological conditions including cloud cover (Pinter \& Jackson, 1985; Asner, 1998). Plant species, maturity, phenology, level of foliage and nutrient status are plant properties affecting reflectance (Asner, 1998; Coops et al., 2003; Gnyp et al., 2014). Geometrical arrangement of objects can affect the spectral reflectance such as leaf orientation, which cannot be controlled during on-line measurements (Asner, 1998; Coops et al., 2003). This creates problems associated with reduced reflection from light scattering. 
Shadows at small scale can be reduced by additional light sources and that opposing lighting can help reduce shadows (Barbedo et al., 2015). Oberti et al. (2014) argued that the angle between the canopy and camera in the range between $0^{\circ}$ to $60^{\circ}$ affects the sensitivity of a mounted on-line (mobile) sensor due to light backscattering, suggesting the potential of an oblique camera angle, to reduce the impact on signal-to-noise ratio (SNR) variation.

A tractor mounted hyperspectral imager allows for on-line field crop canopy sensing and mapping, however, an optimal configuration of the camera, light source and integration time needs to be established for optimal quality of imagery and spectra to be collected. Spectral quality is predominantly affected by sensor integration time, camera orientation, and light height and angle from the object (leaf or canopy). Integration time is the period over which the detector collects photons of light. The greater the integration time and light intensity, the more reflected light is expected to be captured by the detector, providing a higher SNR and pronunciation of the spectral peaks. Though when relying on sun light the intensity can be variable. When applying a spectral technique to a forward moving platform (on-line measurement) longer integration times result in an average spectrum over a larger area, reducing the sensitivity. Furthermore, the greater the distance between the camera and its subject, the larger the area observed and captured by a single pixel, reducing spatial resolution. Therefore, optimising the measurement configuration is essential before on-line field measurements can be successfully carried out. Furthermore, background soil influences canopy spectra, and efforts have been made to remove this influence (Huete, 1988). Based on remote sensing data of the surface soil, Demetriades-Shah et al. (1990) suggested using a second order derivative to remove deviations caused by the soil background. However, none of these studies have investigated the influences of on-line measured (at a depth of 15-20 

canopy spectra.

This paper evaluates, under laboratory conditions, the individual and interaction effects of camera height and angle, integration time and light distance and height on the spectral SNR of a wheat plant canopy captured with a hyperspectral line imager. Furthermore, the influence of on-line measured soil MC and TN on SNR of plant spectra collected on-line in the field is also assessed. This was essential to inform optimal configuration and operational conditions for on-line field measurement of crop canopy and diseases.

Table 1. Factors included in configuring hyperspectral imager (multiple configurations considered)

\begin{tabular}{|c|c|c|c|c|}
\hline $\begin{array}{l}\text { Camera angle, } \\
\operatorname{deg}^{\circ}\end{array}$ & $\begin{array}{l}\text { Light height, } \\
\text { m }\end{array}$ & $\begin{array}{l}\text { Light distance, } \\
\text { m }\end{array}$ & $\begin{array}{l}\text { Camera height, } \\
\text { m }\end{array}$ & $\begin{array}{l}\text { Integration } \\
\text { time, ms }\end{array}$ \\
\hline 0 & 0.90 & 0.60 & 0.15 & 10 \\
\hline 5 & 1.2 & 0.90 & 0.30 & 20 \\
\hline 10 & - & 1.2 & 0.45 & 50 \\
\hline- & - & - & 0.60 & 1000 \\
\hline
\end{tabular}

103

\section{Materials and methods}

\subsection{Hyperspectral configuration in the laboratory}

Winter wheat Triticum Sativum (Solstice variety) was grown outdoors in $600 \times 400 \mathrm{~mm}$ trays (depth of $120 \mathrm{~mm}$ ) with 100 seeds evenly sown and spaced in 5 parallel lines. After seeding the trays were predominantly rain fed, to reduce input of excess salts from treated tap water. 
A push broom hyperspectral imager (spectrograph) (HS spectral camera model from Gilden Photonics Ltd., UK) was used to capture high-resolution line images with a resolution of 1,608 pixels over 1 second, using a diode array detector. It is a 12 bit Basler piA 1600-35 gm camera, with Schneider-Kreuznach XNP1.4/23 lens and has a pixel pitch of $7.4 \mu \mathrm{m}$ interpolated/averaged to $0.6 \mathrm{~nm}$ readings with a spectral range of $400-750 \mathrm{~nm}$. The reflected light from the target travels through the lens, past an entrance slit through a series of available in the market.

The data captured is in the form of a line array, with each pixel containing a spectrum and one detector per pixel across the swath. In order to compile a full image, every line across a means of a dark and white reference, which were collected just before spectral capture, and 
commercially available Spectralon Teflon white calibration panel with $99.9 \%$ white reflectance value.

Factorial analysis was undertaken to understand and quantify the influence of configuration parameters on SNR. The wheat was at growth stage 47 according to Zadoks scale (Zadoks et were conducted in a 9 ha field at Duck End farm, Wilstead, Bedfordshire, UK $\left(52^{\circ} 05^{\prime} 46.3^{\prime \prime} \mathrm{N}\right.$ al., 1974) (whilst the head was booting) at the time of scanning. The studied configuration parameters are shown in Table 1. The same area was scanned in triplicate for the different combination of configurations. The laboratory (simulated-field) measurement configuration is shown in Figure 1. For the indoor environment, two 500 watt diffused broad spectrum halogen lamps were positioned at either end of the crop sample tray. The additional illumination used in the current work was shown by experience to reduce the influence of shadow within the complex and non-homogenous canopy structure. Imagery data was then captured at different camera and light heights, light distances, camera angles and integration times (measured in milliseconds (ms)), as illustrated in Table 1. Light angle was kept constant at $45^{\circ}$, which is debated as the optimal angle to provide the strongest response (Huadong, 2001). Additional opposing lighting was used to reduce shadows (Barbedo et al., 2015). Four integration times of 10, 20, 50 and $1000 \mathrm{~ms}$ were adopted as these cover the most practical ranges. The $1000 \mathrm{~ms}$ integration time illustrates the highest potential time, during which the system will absorb the reflected light hence; this is expected to give the smoothest spectra.

Having determined a suitable configuration in the laboratory the next experiments were designed to apply the configurations to a field environment, and assess the impact of the environmental factors; e.g., soil moisture and total nitrogen, on SNR. Field measurements $\left.0^{\circ} 26^{\prime} 41.4^{\prime \prime} \mathrm{W}\right)$, with an average annual rainfall of $598 \mathrm{~mm}$. The farm has a crop rotation of 


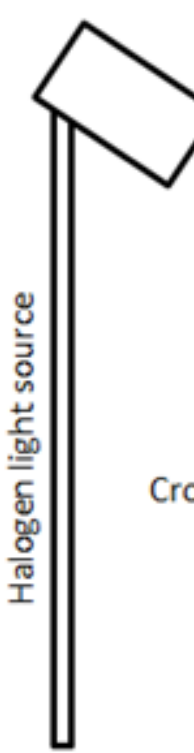

\section{Hyperspectral imager}

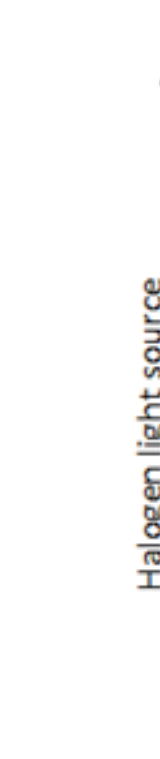

Figure 1. Schematic illustration of the laboratory (simulated field) configurations used and the variables implemented to obtain the hyperspectral data with the highest signal-to-noise ratio (SNR). The hyperspectral imager is a passive sensor, but has been applied with an external halogen light source. The Laser pointers allow to precisely position the hyperspectral imager over the target.

\subsection{On-line soil and crop measurements in the field}

The on-line field measurements included crop spectra and soil MC and TN. The reason why

MC and TN were the selected soil properties, is that the former affects the soil physical and

mechanical conditions, influencing the soil dynamic behaviour below the tractor tyres during the on-line measurement, whereas both may well be linked with crop growth (assuming that TN is directly linked to mineral nitrogen). It is worth mentioning that mineral nitrogen (e.g. nitrate and ammonia) cannot be measured with visible $(400-780 \mathrm{~nm})$ and near infrared $(780-$ 
the potential of the vis-NIR to measure MC and TN, which is attributed to the direct spectral response of these properties in the NIR range (Stenberg et al., 2010; Kuang et al., 2012). The SNR values from the canopies hyperspectral data was compared against the on-line measured soil properties at the same location via Kappa statistics and visual comparisons. This was essential to evaluate whether or not the optimal measurement configuration established in the laboratory is applicable in the field, and whether modifications should be considered.

An on-line vis-NIR soil sensor developed by Mouazen (2006) was used in this study to measure soil MC and TN, with the objective of mapping the spatial variability of these two selected soil properties. The system consists of a subsoiler, opening a smooth trench at $15 \mathrm{~cm}$ depth (Mouazen et al., 2005). The sensor was mounted on a three-point linkage of a tractor travelling at a speed of $3 \mathrm{~km} \mathrm{~h}^{-1}$ and collecting spectral soil data at $10 \mathrm{~m}$ parallel intervals. In order to measure soil spectra an AgroSpec mobile, fibre type, vis-NIR spectrophotometer (Tec5 Technology for Spectroscopy, Oberursel, Germany), with a measurement range of 305$2200 \mathrm{~nm}$ and a light source of $20 \mathrm{~W}$ tungsten halogen lamp were used (Kuang \& Mouazen, 2013). A differential global positioning system (DGPS) (EZ-Guide 250, Trimble, California, USA) recorded the position of the on-line spectra with sub-meter accuracy. The collection of soil spectra and DGPS readings took place at $1 \mathrm{sec}$ sampling resolution using AgroSpec software (Tec5 Technology for Spectroscopy, Oberursel, Germany).

The same hyperspectral imager (Gilden Photonics Ltd, Glasgow, UK) as that used in the laboratory to optimise measurement configuration was used for on-line measurement the wheat canopy in the field. The following hyperspectral measurement configuration was considered: an integration time of $50 \mathrm{~ms}$, a camera height of $0.3 \mathrm{~m}$ and light height and distance of $1.2 \mathrm{~m}$ and a camera angle of $10^{\circ}$. The hyperspectral imager was mounted on a 
tractor boom (Fig. 2) traveling at approximately $4 \mathrm{~km} \mathrm{~h}^{-1}$. The direction and angle of the imager

was kept consistent, and a day with uniformly overcast weather (complete cloud cover) was

selected, which helped prevent issues of moving shadows from lateral sun movement on the data (West et al., 2003). Nevertheless, a handheld LUX meter (RS 180 - 7133, RS Components

\& Controls, India) was utilized to check the sunlight and readings ranged between 1950 and

2000 , indicating no significant difference. The hyperspectral camera was mounted to the side of the tractor. It captures images of 1608 pixels per line, over a one-second interval, which is subsequently logged and geo-located using a DGPS. The collected scans were corrected by means of a dark and a white reference (spectralon $99 \%$ white reflectance panel). The latter

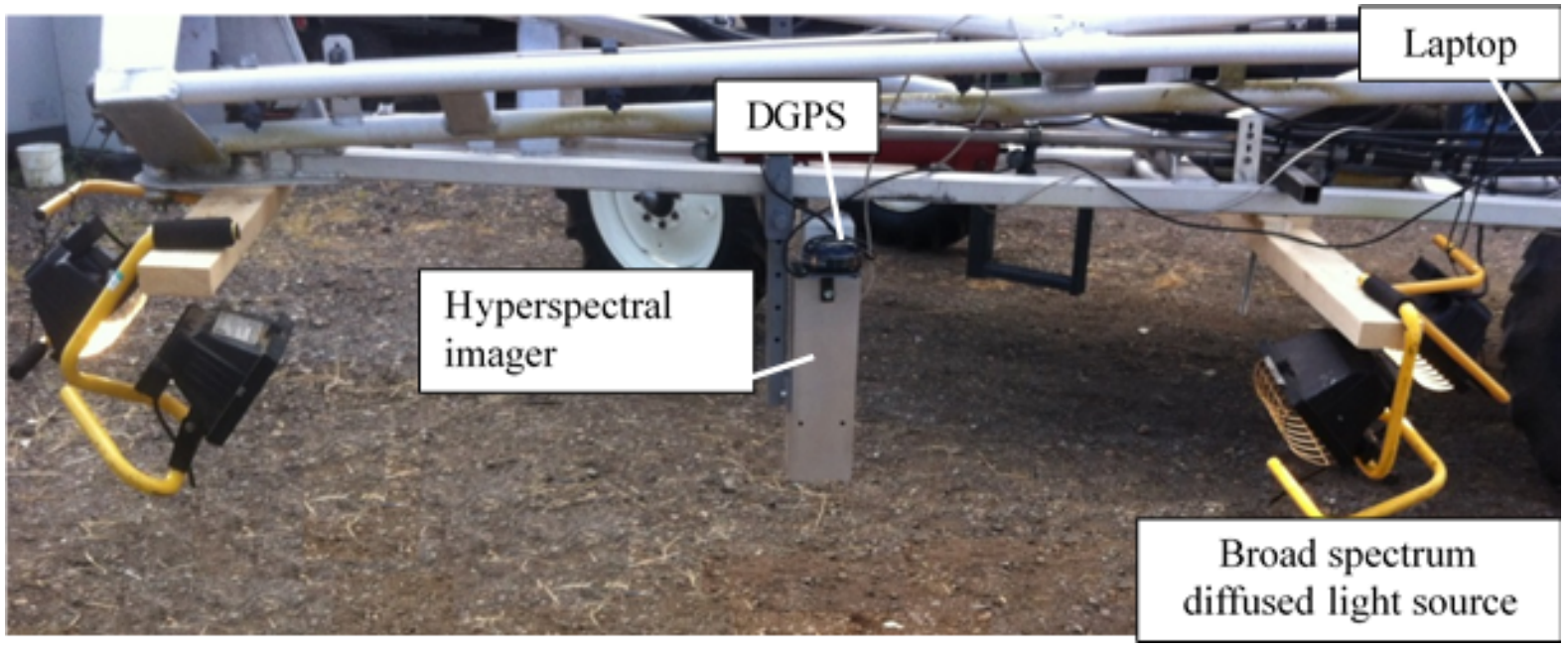

207 Figure 2. Illustrates the on-line field hyperspectral measurement using hyperspectral 208 measurement configuration.

\subsection{Data analyses}

\section{0 \\ 2.1.1 Spectrograph spectral data processing and evaluation}

211 Quality of the wheat canopy spectra (measured both in the laboratory and field) was 212 evaluated by analysing SNR. The SNR is defined here as a ratio of the signal strength to that of 
unwanted interference. A strong signal devoid of interference is the desired outcome in measurements. If the data is too noisy it can hide key features of the spectrum, and data preprocessing such as smoothing can result in them being removed (Dasu \& Johnson, 2003). A noisy spectrum can result in poor calibration models, due to noise being considered as a feature. There are many applications for estimating the SNR from sources such as electrical, chemical, and spectral. Different methods are often applied to estimate the SNR value, depending on the data input. Curran \& Dungan (1989) used a bright homogenous surface to estimate the SNR and produced a method termed the geostatistical method for removal of periodic noise in 
pixels. Spectra of pixels with NDVI values smaller than 0.3 were removed from the calculation

238 of SNR. This method is used by Bravo et al. (2004) and Rouse et al. (1974).

2- Spectra of remaining pixels contain both noise and actual canopy signal, the intensity of which depends on the pixel position within a complex canopy structure of the crop. This will allows mimicking the measurement of actual canopy.

3- Variation in position of pixels for a series of scans can be minimised by fixing the position of crop trays, so that line images are collected from the same target area for different measurement configurations. In this case, whilst uniform intensity cannot be achieved across the spectrum in one scan, each pixel has almost the same target object throughout all the scans.

The calculation of the SNR was done in this study following a similar approach adopted by Ramamurthy et al. (2014) and described earlier by Smith (1999). As the data collected is single line 2-dimensional captures, the data was assessed as spectra rather than images. This method of SNR was selected as it could be used as a basis of comparison between individual spectral data captures. We calculated the SNR of individual wavelength $\left(S_{N} R_{w}\right)$ as follows:

Where: $M_{w}$ is the mean reflectance value of individual wavelength through all the pixels, and $\mathrm{SD}_{\mathrm{w}}$ is the standard deviation of individual wavelength of all pixels (Fig. 3). It is worth noting that all pixels were considered important in the current work to calculate SNR. An alternative 
approach to this method would have been to select central pixels only to calculate SNR, a method applied by Daumard et al. (2010).

The mean spectral signal describes what is being measured, whereas the standard deviation represents noise and other interference for each pixel (Smith, 1999). $M_{w}$ values for different wavelengths were calculated on remaining 967 wavebands, after removing the spectral range outside of the 400 to $750 \mathrm{~nm}$ range, since they were found to be noisy. Once Mw and SDw are calculated for each individual wavelength, the SNR for a spectrum $\left(\mathrm{SNR}_{s}\right)$ was calculated as follows (see Fig. 3):

$\mathrm{SNR}_{\mathrm{s}}=\mathrm{M}_{\mathrm{s}} / \mathrm{SD}_{\mathrm{s}}$

Where: $M_{s}$ is mean reflectance of all wavelengths in a spectrum (a scan) and $S_{s}$ is mean standard deviation of all wavelengths in a spectrum.

The SNRs was used in this study to evaluate the strength of scans, hence, the quality of spectral signal. It is worth noting that SD is not important in itself, but only in comparison to the mean. While convenient and straightforward, the deviation is a statistic doesn't fit well with the physics of how signals operate (Smith, 1999). Therefore, a strong SNR with pronounced absorption peaks provides increased recognition for the association of spectral signatures to a subject.

A principal component analysis (PCA) was also undertaken on laboratory measured hyperspectral data only using Statistica software (StatSoft inc., Oklahoma USA) to identify 
parameters with the greatest impact on SNR. PCA is a statistical method, which analyses the distribution of data in multidimensional space (principal components) or similarity maps, where similarities (within groups of variable) and differences between groups can be evaluated (Dytham, 2011). In this instance, SNR, integration time, light height and distance, and camera angle and height were used as input variables for the PCA analysis. Additionally, a two-way analysis of variance (ANOVA) was carried out with RStudio software (RStudio Boston, MA) to estimate significant influences of individual variables and interaction between variables on SNR (Webster, 2007; Dytham, 2011).

\subsubsection{On-line soil sensor calibration}

Laboratory measurements of MC and TN were carried out using standard reference method. Soil MC was measured with oven drying of samples at $105^{\circ} \mathrm{C}$ for $24 \mathrm{~h}$, whereas TN was measured with a TrusSpecCNS spectrometer (LECO Corporation, St. Joseph, MI, USA), using the Dumas combustion method (Dumas, 1826 as cited by Buckee, 1994). The on-line collected soil spectra were subjected to pre-processing before modelling. Pre-processing included noise cut by removing wavelengths smaller than $400 \mathrm{~nm}$ and larger than $1900 \mathrm{~nm}$. Noise cut was followed successively by maximum normalisation, first derivative and smoothing. Partial least squares regression (PLSR) analysis with leave-one-out full cross-validation was carried out to establish correlations between soil spectra and laboratory measured MC and TN. Spectra preprocessing and PLSR analysis were carried out using Unscrambler 7.8 software (Camo Inc.; Oslo, Norway). 


\subsection{Mapping}

302 Maps for on-line vis-NIR predicted MC and TN and on-line spectrograph measured crop canopy of wheat were developed used ArcGIS 10 (ESRI, California, USA) software. Kriging was used to develop maps, assuming that the distance or direction between sample points reflects a spatial correlation that can be used to explain spatial variations. The advanced parameters option in ArcGIS 10 software (ESRI, California, USA) allowed control of the semi-variogram used for kriging, selecting spherical as the best fit. The semi-variogram values were calculated in RStudio (RStudio, Boston, MA).

The similarity assessment between maps can be performed by visual inspection and statistical tests (Tekin et al., 2013). The simplest way of comparing between maps is by visual inspection, to conclude on similarities that may exist or not. However, this is insufficient, as quantitative estimation of similarity is a more robust approach to adopt. To compare statistical relationship of pairs of maps, Kappa statistics (Cohen, 1960) analyses were performed to calculate Kappa value (к), using SPSS (Statistical Package for the Social Sciences, IBM, Armonk, New York, USA). However, before running Kappa statistics data was subjected to raster analyses to have the same $5 \mathrm{~m}$ by $5 \mathrm{~m}$ grid size for all maps, after which the data was normalised. 


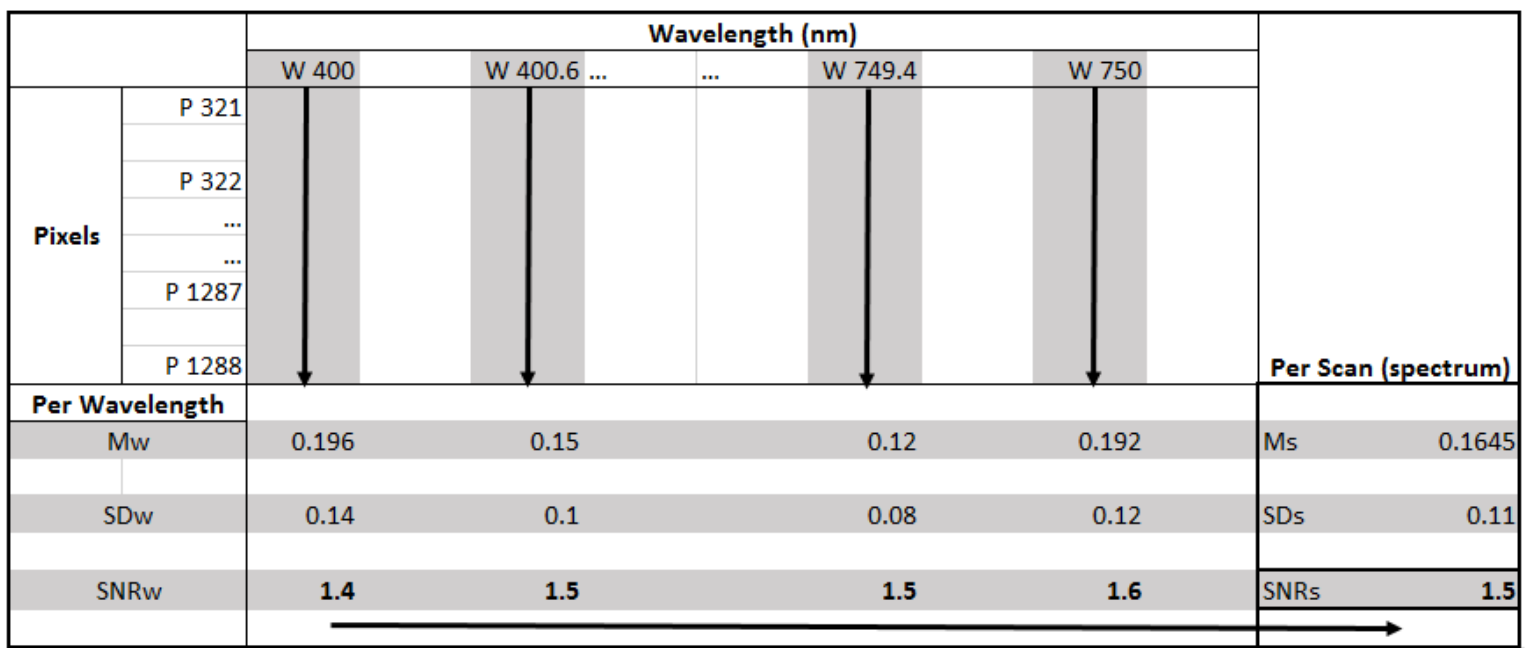

319 Figure 3. Schematic illustration outlining how mean, standard deviation and signal-to-noise 320 ratio for wavelength $\left(\mathrm{M}_{w}, \mathrm{SD}_{\mathrm{w}}, \mathrm{SNR}_{\mathrm{w}}\right.$, respectively) and a spectrum $\left(\mathrm{M}_{s}, \mathrm{SD}_{\mathrm{s}}, \mathrm{SNRs}\right.$, 321 respectively) were calculated.

\section{Results and Discussion}

\subsection{Spectral quality in the laboratory}

324 Typical crop canopy spectra can be observed in Figure 4, which shows clear, noisy and saturated spectra. The clear and saturated spectra can be observed to be more pronounced, whereas a weak absorption in the spectral signature and interference in the noisy spectrum leads to reduced quality, low SNR, masking detail in the signature and causing a loss of important spectral information through the entire spectral range studied. The clear spectrum is the best quality, and the target to be obtained. The noisy spectrum is caused by the low integration times, and greater distance of the Halogen light source. A strong SNR with pronounced absorption peaks would allow for a greater success in analysis of crop assessments and disease presence. Although, pre-processing of spectral data includes techniques such as smoothing, if the process of cleaning the data is intensive due to noisy spectra it can lead to the loss of important spectral features, and thus impact on the success of analysis (Dasu \& Johnson, 2003). 
Saturation predominantly occurs within the central pixels associated with the highest reflectance, causing data in the peaks of the electromagnetic spectrum to be lost (starting around $650 \mathrm{~nm}$ ). Leaf reflectance in the NIR range is affected by the structure of the plant leaves (Gates et al., 1965), and can be related to the leaf wax coating (Cameron 1970). In the case of spectral saturation the data becomes unusable. In the remaining parts of the spectrum, however, particularly the visible range $(400-700 \mathrm{~nm})$, there is a lower reflectance due to a larger absorption of the light, which is attributed to the photosynthetic pigments of the plants leaves, (Gates et al., 1965).

When analysing the entire spectral signature saturation causes a reduction in sensitivity.

Therefore, sensor configurations leading to saturated spectral data were removed from the analysis of SNR. All saturated data was obtained with integration time of $1000 \mathrm{~ms}$, this is in line with the literature, where larger integration times can cause saturation of the data (Dell'Endice, 2008).

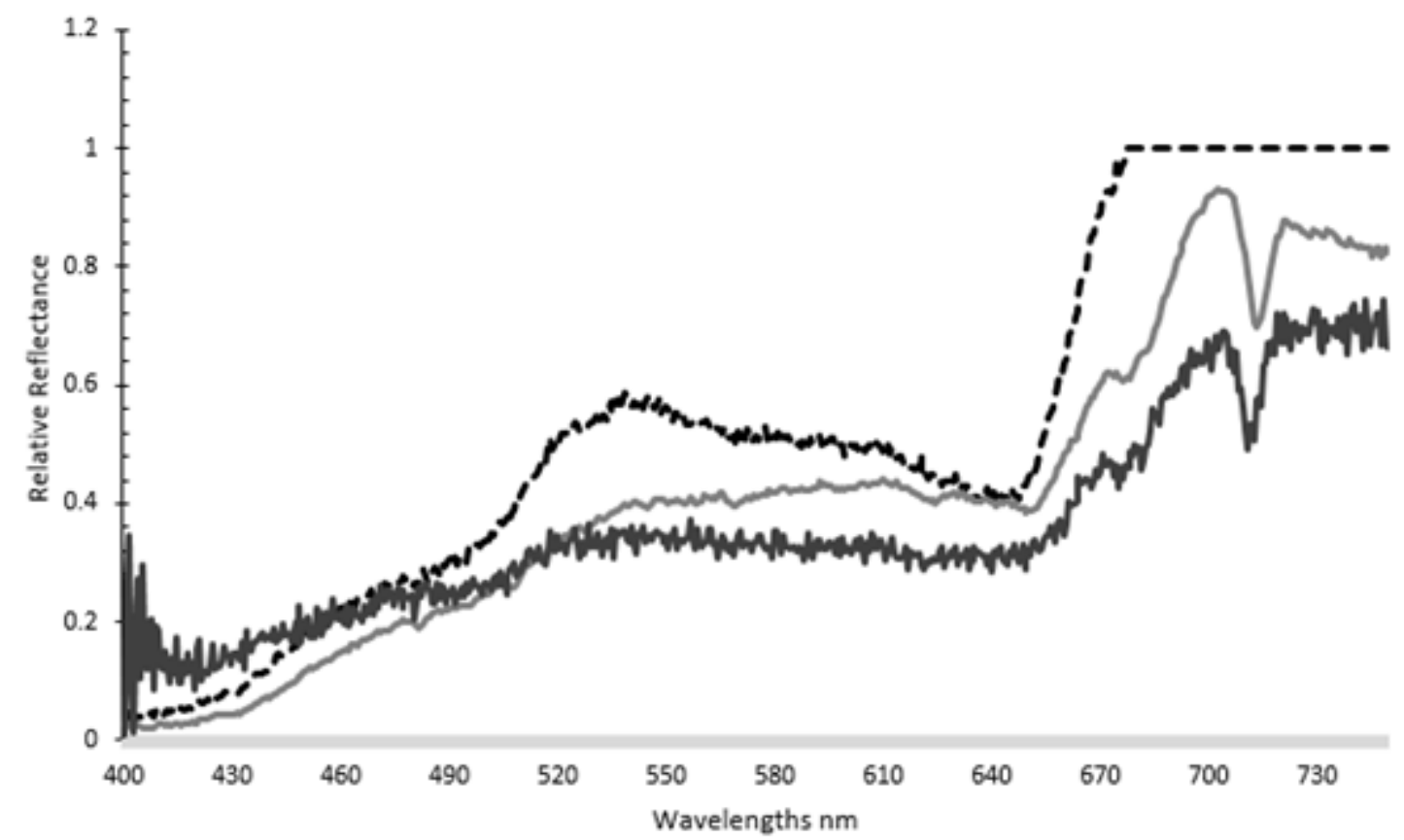
black line with 1.2 SNR), and saturated (the dotted line with 1.8 SNR) spectra of wheat canopy. 
The saturated spectrum flattens off at $680-750 \mathrm{~nm}$ and important information is lost on the peak around $700 \mathrm{~nm}$ that is otherwise illustrated by the clear spectra.

\subsection{Hyperspectral images configuration parameters; laboratory}

The integration time considerably affected the SNR, expressed as average and SD values (Eq. 1), as shown in Table 2. The longer the integration time the greater the opportunity for more energy to be captured by the spectrograph. The $1000 \mathrm{~ms}$ configuration was the highest potential integration time and was trialled (under field simulations) to see the potential highest SNR, whilst the manufacturer recommended around $20 \mathrm{~ms}$ for laboratory use. The results from the 1000 ms integration time provided a high percentage of saturated results, the non-saturated recordings were of the same SNR values to that of the 50 ms integration times, so integration times higher than $50 \mathrm{~ms}$ were not trialled further. With varying the integration time, parameters have constant values apart from the camera height. The optimal camera height decreases with the integration time, the closer the camera to the object the shorter the integration time required. This may mean that the most influencing parameters on the SNR are the integration time and camera height. Other studies demonstrated that the movement (adjustments, bounce or vibration) between the imager and the subject during integration time can cause a compiled image to be warped or noisy (Zhong et al., 2011). For on-line measurements the easiest variable to control is integration time, as angle and height can alter slightly as ground is uneven, crop stands vary and unavoidable movement in mountings. It becomes necessary to have the optimal configurations set initially but to understand there would be slight deviations. A NADIR camera angle was selected, due to reports from Oberti et al. (2014) and Van Beek et al. (2013).

ANOVA showed that all individual variables and interactions of variables have significant influences on SNR at the (0.005) 95\% confidence level, except for the interactions of light 
distance and integration time (0.001), and light distance, camera height and integration time (0.01). Therefore, the null hypothesise 'that variables and subsequent interactions of variables will have no significant effect on SNR' can be rejected.

Figure 5 illustrates the similarity maps of principal component (PC) 1 and 2 (a), and PC1 and 3 (b) accounting for $47.62 \%$ and $42.26 \%$ of variance, respectively. Examining the plot of PC1 vs PC2 one can observe that the integration time, SNR and camera height are gathered in one group, which explains these to be closely related. Integration time shows the strongest influence and correlation with SNR, whereas, camera height demonstrates the second closest corresponding variable on SNR with the latter having a weaker influence (Fig. 5a). Light distance, light height and camera angle seem to have only minor influences on SNR, as they make a separate group associated with PC2 with minor variance associated with PC1. Disregarding other variables, it becomes clear that a longer integration time and a smaller camera height result in a higher SNR.

Although the light distance and height are strongly associated in the PCA similarity maps (Fig. $5 a \& b)$, they have a negligible influence on the SNR. This does not mean that the absence of light would have no effect. As long as there was ample diffused light, in order for the detector to collect photons, light variables appeared to have little impact. Although the influence of camera height was the second largest in the PC1-PC2 similarity map, in the PC1-PC3 similarity map, the camera height has small influence on SNR similar to the light variables. Furthermore, the close correlation between camera height and integration time shown in the PC1-PC2 similarity map is not observed in the PC1-PC3 similarity map. In the latter similarity map (Fig. $5 b)$, the camera angle has the second largest influence on SNR after the integration time. This means that both camera height and angle have strong influences on the SNR. Under 
laboratory measurement conditions, larger camera angles were reported to be beneficial for powdery mildew recognition in leaves of grape vines by Oberti et al. (2014). Smaller view angles are discussed by Pisek et al. (2009) to theoretically be better due to observing a larger area. Rautiainen et al. (2008) concluded greater nadir angles are more suitable for viewing the top canopy and had a very limited view of the understory. This is supported by the finding that $80 \%$ of the crop yield is calculated from the health of the top 3 leaves (HGCA, 2008). The correlation between green leaf retention and yield has been observed in a number of trials (Reynolds et al., 2009; Ali et al., 2010; Hunt \& Poole, 2010) and can be observed with reference to trial work in barley conducted by the authors in 2009 , where every $1 \%$ reduction in green leaf area on flag-1 at GS80 correlated to a $20 \mathrm{~kg} / \mathrm{ha}$ loss in yield. This is true when applying a camera angle. But when assessing light conditions, an off-nadir angle can create more lighting variability, due to shady and sunlit areas of the crop. Van Beek et al. (2013)

411 found that for smaller off-nadir viewing angles $\left(<20^{\circ}\right)$ of the sensor, the sun orientation was

412 found not to be important. This, along with issues of shadow from the infield mountings, is why small angles for the configurations were selected. 


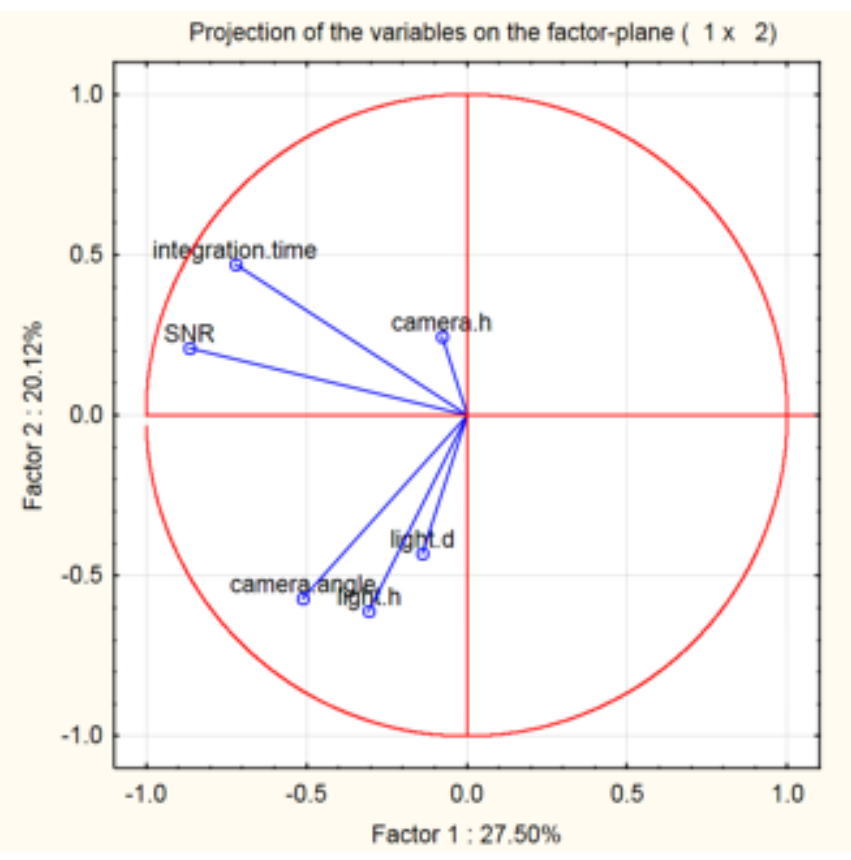

(a)

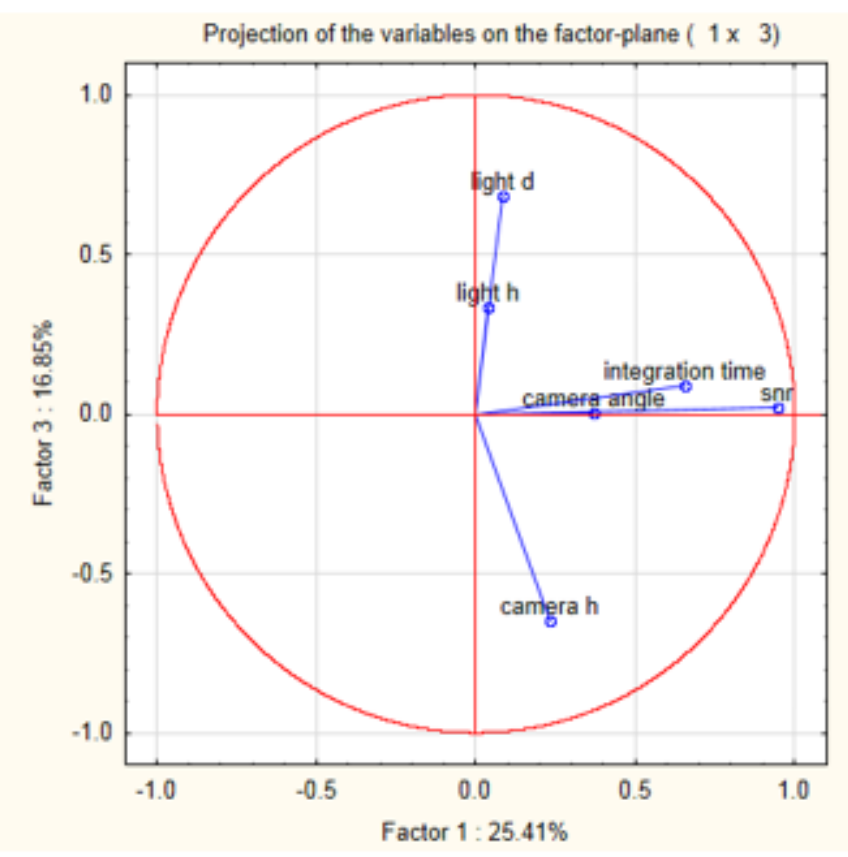

(b)

415 Figure 5. Principal component analysis similarity maps developed for principal components 1 416 and 2 (a) and for principal components 1 and 3 (b). Input variables are camera and light height, 417 light distance, camera angle, integration time and signal-to-noise ratio (SNR) 
419 To make a decision on an optimal configuration that would result in the highest SNR, was not a straightforward process. However, when SNR values are arranged according to the integration time (Table 2), a clear trend is observed. For example, SNR increased with integration time, where the highest values of SNR were obtained with an integration time of $1000 \mathrm{~ms}$, however, they were only marginally higher than the SNR values at 50ms. Since several variable combinations resulted in similar SNR values, it is perhaps premature to suggest the optimal configuration as the single highest, so configurations with SNR variability of less than $5 \%$ from the highest reading (of each integration time) were considered for further evaluation. The further analysis confirms that the highest SNR occurs with the same configurations of $1.2 \mathrm{~m}, 1.2 \mathrm{~m}$, and $10^{\circ}$ of light height, light distance and camera angle, respectively. As stated earlier, the camera height is negatively correlated with the integration time (Table 2). A possible theory for reoccurrence of these configurations could be that they allow for the greatest amount of reflected light to be captured by the detector. Among the integration time steps of 10, 20, 50 and 1000 ms selected, a practical range for on-line measurement is between $10 \mathrm{~ms}$ and $50 \mathrm{~ms}$, a range which is suggested by the manufacturers. Assuming that the best integration time for practical on-line (mobile) measurement in the field is $50 \mathrm{~ms}$, the optimal configuration parameters of $1.2 \mathrm{~m}, 1.2 \mathrm{~m}, 0.3 \mathrm{~m}$ and $10^{\circ}$ are recommended for light height, light distance, camera height, and camera angle, respectively.

437 The average SNR for this integration time is 1.669 (seen in table 2), which in reference to Smith (1999) we believe is a strong signal for a crop canopy. These optimal configuration parameters were adopted for the on-line measurement of wheat canopy measurement in this study. 
443 Table 2. Average and standard deviation (SD) values of the highest signal-to-noise-ratio

444 (SNR) obtained with different integration time, camera and light source settings when

445 scanning a wheat canopy. Theoretical forward distance travelled (and captured to a single

446 data line) if applied on a moving platform at field scale.

447

\begin{tabular}{llllllll}
\hline Average, & SD & Integration & Light & Light & Camera & Camera & Distance \\
SNR & time, & height, & distance, & height, & angle, & travelled, \\
& & $\mathbf{m s}$ & $\mathbf{m}$ & $\mathbf{m}$ & $\mathbf{m}$ & $\mathbf{d e g}$ & $\mathbf{m}$ \\
\hline 1.688 & 0.102 & 1000 & 1.2 & 1.2 & 0.15 & 10 & 4 \\
1.669 & 0.160 & 50 & 1.2 & 1.2 & 0.30 & 10 & 0.2 \\
1.471 & 0.103 & 20 & 1.2 & 1.2 & 0.45 & 10 & 0.08 \\
1.386 & 0.078 & 10 & 1.2 & 1.2 & 0.45 & 10 & 0.04
\end{tabular}

\section{$448 \quad 3.3$ Hyperspectral imager; on-line measurement}

449 During the on-line measurement, it was noticed that there was an unavoidable bounce in the

450 boom observed to be at $\pm 0.2 \mathrm{~m}$ of the original height of the mounting (set at $0.3 \mathrm{~m}$ above the

451 crop canopy). On inspection of the data the camera height from the object affected the

452 uniformity of light intensity measured across the pixels, (particularly at the beginning and end

453 of the captured line). Therefore, for calibrating the on-line hyperspectral scans, it is

454 recommended to overcome this fluctuation by removing the first and last 320 pixels from the

455 spectral data. This is specific to this hyperspectral imager but is an interesting factor for

456 consideration.

457 Comparing the laboratory with on-line field measured canopy spectra, one can observe that

458 the laboratory reflectance scans to be marginally higher and more consistent than the on-line

459 scans (Fig. 6). Initially, the on-line spectra showed variation in canopy spectra throughout the

460 field, which appeared to be in response to different crop spatial conditions. For example, the

461 field scan 1 refers to canopy of water stressed wheat plants, whereas field scan 2 refers to 


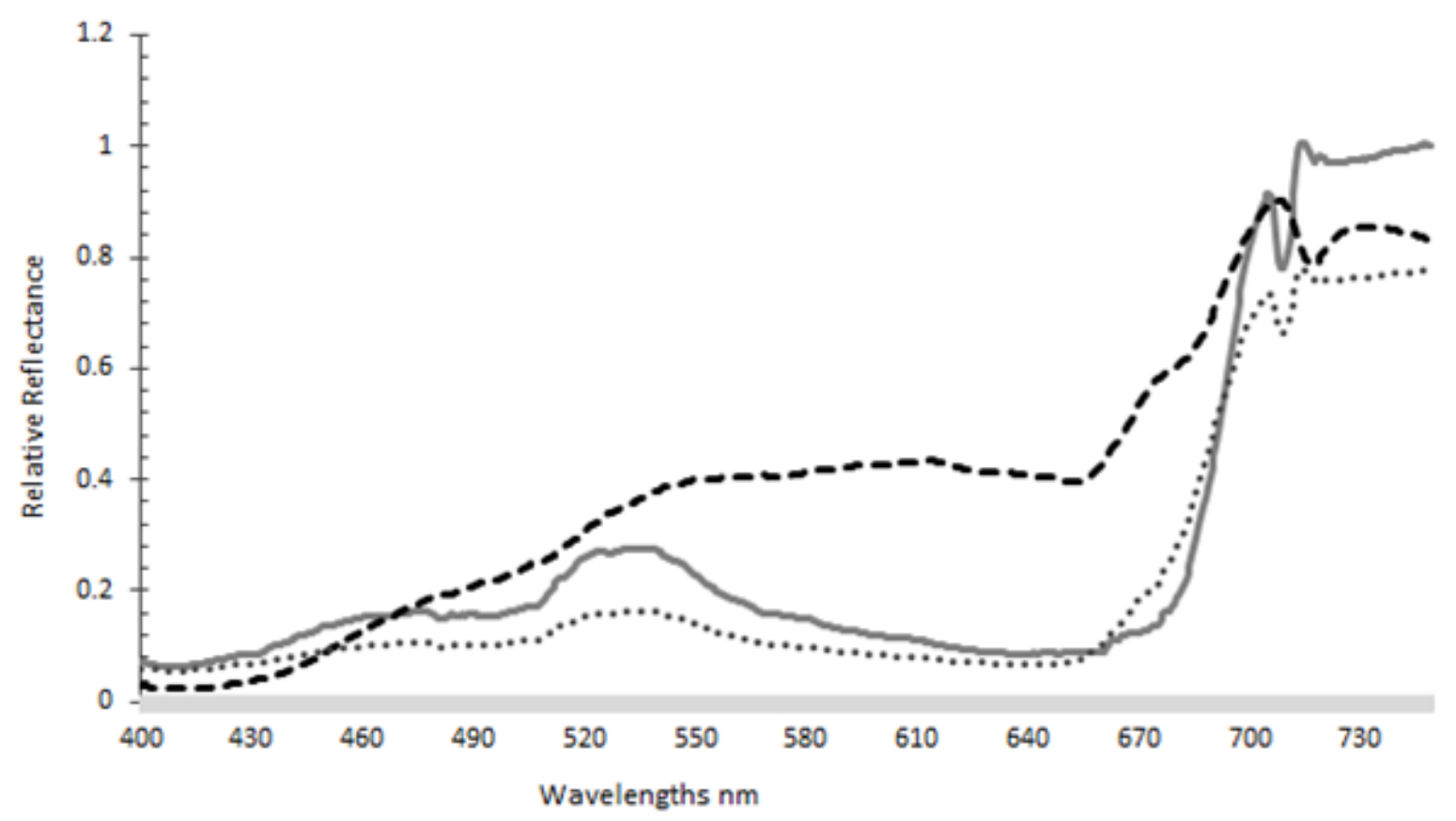

467 Figure 6. Comparison of canopy spectra of wheat crop obtained in the laboratory (the dashed 468 line) and on-line in the field (the dotted and grey lines). Crop was of the same culture and at 469 comparable growth stages. Laboratory scans were collected under the suggested optimal 470 configurations. The on-line field scans are: 1 ) field scan 1 (the grey line) is of a more water stressed plant and 2) field scan 2 (the dotted line) is of a less water stressed plant. 

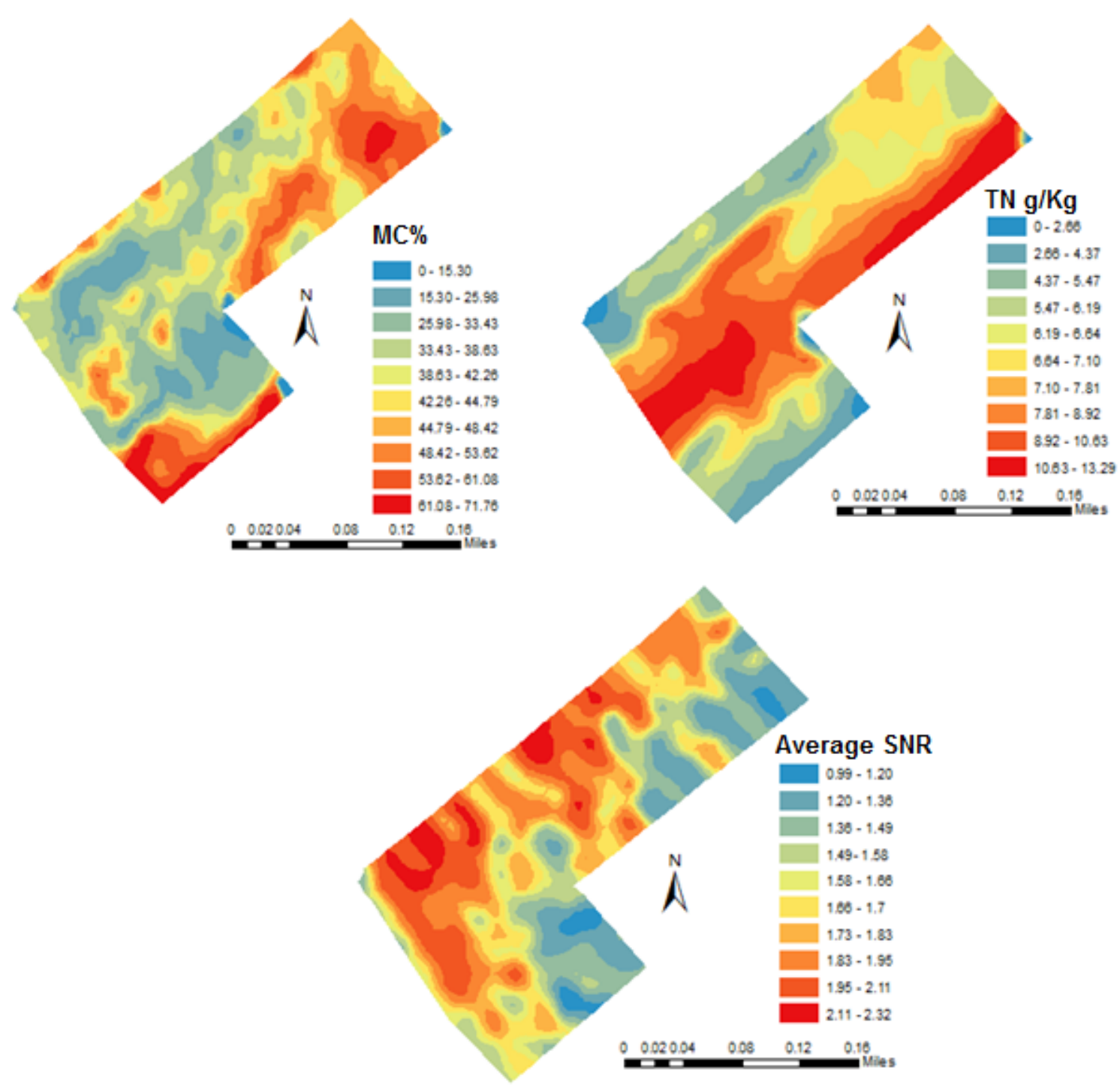

474 Figure 7. Maps of on-line measured soil moisture content (MC) (a) total nitrogen (TN) (b), and 475 the average signal-to-noise ratio (SNR) per scan (c). measurement The on-line measured soil MC and TN maps of the field provided a visual explanation (Fig. 7)

480 for a drop in absorbance within certain areas of the field. Comparing the MC map with the SNR map, one can draw a general conclusion that areas of low MC correspond with areas of 
high SNR, and vice versa (areas of high MC being of low SNR). This can be explained by the fact that soil deformation under the tractor tyre in wet soils is larger than that in drier soil conditions. A larger soil deformation should result in a larger fluctuation in camera height and angle, compared to the baseline setup, which may lead to reducing the SNR. Work carried out by Söhne (1958) showed that an increase in the MC of a soil and the increase in payload on a tyre both increase the depth of soil deformation.

Both on-line measured MC and TN were found to have significant effects on the SNR of the wheat canopy spectra at 95\% confidence. The kappa values between the SNR map on one hand and TN and MC maps on the other hand confirm spatial similarity or difference the field. Landis \& Koch (1977) classified Kappa values into different categories: 0-0.20, 0.21-0.40, $0.41-0.60,0.61-0.80$, and $0.81-1$, which indicate slight, fair, moderate, substantial, and almost perfect agreement, respectively. Results show that the Kappa value between TN and SNR is rather small (kappa $=0.56)$, indicating moderate similarity between these two maps. Since TN is the main source of mineral nitrogen essential for crop growth and development, it can be influential on the quality of crop canopy and SNR. The kappa value between MC and SNR was much higher (kappa $=0.75$ ) than that between TN and SNR map, confirming substantial similarity between the two maps. This supports the earlier suggestion about the influence of $M C$ on soil deformation that changes the initial (optimal) hyperspectral measurement configuration obtained in the laboratory and implemented for on-line measurement in the field. In order to reduce the negative influence of soil deformation on SNR due to high soil MC during on-line scanning, proper spectra pre-processing is recommended e.g., normalisation, derivation, and multiplicative scatter corrections. Further research is required to confirm this assumption, as when measuring on-line, deviations from 
the optimum configuration (due to tractor vibration, bounce in the boom and soil deformation) were unavoidable.

\section{Conclusions}

This study was undertaken to determine an optimal measurement configuration of a hyperspectral line imager $(400-750 \mathrm{~nm})$ by evaluating the individual and interaction effects of the systems configurations, on the hyperspectral calculated SNR for measurement of a wheat canopy under laboratory scanning conditions. Optimal configuration was determined and implemented for on-line field measurement. The influence of on-line measured soil moisture content (MC) and total nitrogen (TN) on SNR was evaluated. Results allowed the following conclusions to be drawn:

1- The integration time followed by the camera height and camera angle appeared to have the largest influence of the SNR. A long integration time (>50 ms) was of a negligible influence and only slightly increased the SNR, but result in spectral saturation, hence should be avoided.

3- The PCA similarity map showed that the light height and distance have a strong correlation with each other but a minimal influence on SNR.

4- Both on-line measured MC and TN were found to have significant effects on the SNR of the wheat canopy spectra at $95 \%$ confidence. The on-line soil measurements revealed stronger spatial similarity between the hyperspectral SNR and MC maps (kappa value $=0.75$ ), which was attributed to soil deformation below the tractor tyre.

5- The variable reflected light intensity captured by the different pixels across the line imagery is an interesting factor to take into account, due to the impact of varying camera height during 
527 the on-line measurement. Whilst the solution suggested here is appropriate, it is camera 528 specific.

529 Further work is planned to overcome variation in SNR associated with camera height changes

530 (vibration, bounce in the boom, and soil deformation during the on-line measurement) by the 531 implementation of a proper spectra pre-processing. It is also planned to implement these 532 hyperspectral measurement configurations for on-line measurements of crop canopy for 533 detection of crop health and disease presence.

\section{Acknowledgement}

We acknowledge the funding received for FarmFUSE project from the ICT-AGRI under the

European Commission's ERA-NET scheme under the 7th Framework Programme, and the UK

Department of Environment, Food and Rural Affairs (contract no: IF0208).

\section{References}

541 Ali M.A., Hussain M., Khan M.I., Ali Z., Zulkiffal M., Anwar J., Sabir W., Zeeshan M. (2010).

542 Source-sink relationship between photosynthetic organs and grain yield attributes during 543 grain filling stage in spring wheat (Triticum aestivum). International Journal of Agriculture and 544 Biology, 12: pp. 509-515.

545 Asner, G. P. (1998). Biophysical and biochemical sources of variability in canopy reflectance. 546 Remote sensing of Environment, 64(3), 234-253. 
547 Barbedo, J. G., Tibola, C. S., \& Fernandes, J. M. (2015). Detecting Fusarium head blight in 548 wheat kernels using hyperspectral imaging. Biosystems Engineering, 131, 65-76.

549 Bock, C. H., Graham, J. H., Gottwald, T. R., Cook, A. Z., \& Parker, P. E. (2010). Wind speed 550 effects on the quantity of Xanthomonas citri subsp. citri dispersed downwind from canopies 551 of grapefruit trees infected with citrus canker. Plant Disease, 94(6), 725-736. Foliar disease detection in the field using optical sensor fusion. Agricultural Engineering International: CIGR Journal of Scientific Research and Development. Manuscript FP 04 008. Vol.6, $14 p$.

Buckee, G. K. (1994). Determination of total nitrogen in barley, malt and beer by Kjeldahl

557 procedures and the Dumas combustion method - Collaborative trial. Journal of the Institute 558 of Brewing 1994, 100 (2) pp. 57-64.

Cameron, R. J. (1970). Light intensity and the growth of Eucalyptus seedlings: II. The effect of cuticular waxes on light absorption in leaves of Eucalyptus species. Australian Journal of Botany, 18: pp. $275-284$.

Cohen, J., 1960. Kappa: Coefficient of concordance. Educational Psychologist, Measurement, 20, p.37

Coops, N. C., Smith, M. L., Martin, M. E., \& Ollinger, S. V. (2003). Prediction of eucalypt foliage 565 nitrogen content from satellite-derived hyperspectral data. IEEE Transactions on Geoscience and Remote Sensing, 41(6), 1338-1346. 
Curran, P.J. and Dungan, J.L., 1989. Estimation of signal-to-noise: a new procedure applied to AVIRIS data. IEEE Transactions on Geoscience and Remote Sensing, 27(5): pp. 620-628.

Dasu, T., \& Johnson, T. (2003). Exploratory data mining and data cleaning (Vol. 479). John Wiley \& Sons.

Daumard, F., Champagne, S., Fournier, A., Goulas, Y., Ounis, A., Hanocq, J.F. and Moya, I., 2010. A field platform for continuous measurement of canopy fluorescence. IEEE Transactions on Geoscience and Remote Sensing, 48(9), pp.3358-3368.

574 Dell'Endice, F. (2008). Improving the Performance of Hyperspectral Pushbroom Imaging Spectrometers for Specific Science Applications. In ISPRS 2008: Proceedings of the XXI Congress: Silk Road for Information from Imagery: The International Society for Photogrammetry and Remote Sensing (pp. 215-220).

Demetriades-Shah, T.H., Steven, M.D. and Clark, J.A., 1990. High resolution derivative spectra in remote sensing. Remote Sensing of Environment, 33(1): pp.55-64.

Dytham, C. (2011). Choosing and using statistics: a biologist's guide. John Wiley \& Sons.

Gates, D.M., Keegan, H.J., Schleter, J.C. and Weidner, V.R. (1965). Spectral properties of plants. Applied optics, 4(1), pp.11-20.

Gnyp, M.L., Miao, Y., Yuan, F., Ustin, S.L., Yu, K., Yao, Y., Huang, S. and Bareth, G. (2014). Hyperspectral canopy sensing of paddy rice aboveground biomass at different growth stages. Field Crops Research, 155: pp. 42-55. 
588

589

590

591

592

593

594

595

596

597

598

599

600

601

602

603

604

605

606

607

oat and canary grass in wheat fields for less herbicide application. Agronomy for Sustainable Development, 30(3), pp. 689-699.

Hahn, F., (2009). Actual pathogen detection: Sensors and algorithms-a review. Algorithms 2, $301-338$.

Herrmann, I., Shapira, U., Kinast, S., Karnieli, A. and Bonfil, D.J., 2013. Ground-level hyperspectral imagery for detecting weeds in wheat fields. Precision agriculture, 14(6): pp. 637-659.

Home Grown Cereal Authority (HGCA) (2008). The spring wheat disease management guide. Warwickshire: Home Grown Cereals Authority (HGCA Publication).

Huadong, G. (2001). Applications of Radar Remote Sensing in China. New York: Taylor and Francis inc. 56.

Huete, A. R. (1988). A soil vegetation adjusted index (SAVI). Remote Sensing of Environment, 25: pp. $295-309$.

Hunt J., \& Poole N. (2010). Simulating leaf area duration to predict yield response to foliar fungicide in wheat and barley. In: Dove H, Culvenor RA, editors. Food security from. sustainable agriculture. Proceedings of 15th Agronomy Conference; 2010 Nov; Lincoln, New Zealand.

Kuang, B., Mahmood, H.S., Quraishi, M.Z., Hoogmoed, W.B., Mouazen, A.M. \& van Henten, E.J. (2012). 4 Sensing Soil Properties in the Laboratory, In Situ, and On-Line: A Review. Advances in Agronomy, 114(1), pp.155-223. 
608

609

610

611

612

613

614

615

616

617

618

619

620

621

622

623

624

625

626

627

628

Kuang, B. \& Mouazen, A.M., (2013). Non-biased prediction of soil organic carbon and total nitrogen with vis-NIR spectroscopy, as affected by soil moisture content and texture. Biosystems engineering, 114(3), pp.249-258.

Landis, J. R., \& Koch, G. G. (1977). The measurement of observer agreement for categorical data. Biometrics, 33, 159-174.

Lee, J. B., Woodhyatt, S., and Berman, M. (1990). Enhancement of high spectral resolution remote-sensing data by a noise-adjusted principal components transform. IEEE Transactions on Geoscience and Remote Sensing, 28: pp. 295-304

Lenk, S., Chaerle, L., Pfündel, E.E., Langsdorf, G., Hagenbeek, D., Lichtenthaler, H.K., Van Der Straeten, D., \& Buschmann, C., (2007). Multispectral fluorescence and reflectance imaging at the leaf level and its possible applications. Journal of Experimental Botany 58 (4), 807-814.

López, M.M., Bertolini, E., Olmos, A., Caruso, P., Gorris, M.T., Llop, P., Penyalver, R., \& Cambra, M., (2003). Innovative tools for detection of plant pathogenic viruses and bacteria. International Microbiology 6, 233-243.

Moshou, D., Bravo, C., Oberti, R., West, J., Bodria, L., McCartney, A., \& Ramon, H., (2005). Plant disease detection based on data fusion of hyper-spectral and multi-spectral fluorescence imaging using Kohonen maps. Real-Time Imaging 11 (2), 75- 83.

Mouazen, A. M. (2006). Soil Survey Device. International publication published under the patent cooperation treaty (PCT). World Intellectual Property Organization, International Bureau. International Publication Number: WO2006/015463; PCT/BE2005/000129; IPC: G01N21/00; G01N21/00. 
629

630

631

632

633

634

635

636

637

638

639

640

641

642

643

644

645

646

647

648

649

650

Mouazen, A.M.; Anthonis, J.; \& Ramon, H., (2005). An automatic depth control system for online measurement of spatial variation in soil compaction, Part 4: improvement of compaction maps by using a proportional integrative derivative depth controller. Biosystems Engineering, 90(4), 409-418.

Oberti, R., Marchi, M., Tirelli, P., Calcante, A., Iriti, M., \& Borghese, A. N. (2014). Automatic detection of powdery mildew on grapevine leaves by image analysis: Optimal view-angle range to increase the sensitivity. Computers and Electronics in Agriculture, 104, 1-8.

Okamoto, H. \& Lee, W.S. (2009). Green citrus detection using hyperspectral imaging. Computers and Electronics in Agriculture, 66(2): pp.201-208.

Pinter, JR, P.J. and Jackson, R.D. (1985). Sun-angle and canopy-architecture effects on the spectral reflectance of six wheat cultivars. INT. J. REMOTE SENSING, 6(12): pp. 1813-1825.

Pisek, J., Chen, J. M., Miller, J. R., Freemantle, J. R., Peltoniemi, J. I., \& Simic, A., (2009). Mapping forest background reflectance in a boreal region using multi-angle Compact Airborne Spectrographic Imager (CASI) data. IEEE Transactions on Geoscience and Remote Sensing, 47. doi:10.1109/TGRS.2009.2024756.

Ramamurthy, R., Canning, C. F., Scheetz, J. P., \& Farman, A. G. (2004). Impact of ambient lighting intensity and duration on the signal-to-noise ratio of images from photostimulable phosphor plates processed using DenOptix ${ }^{\circledR}$ and $\operatorname{Scan}^{\circledR}$ systems. Dentomaxillofac Radiol, 33(5), 307-11

Rautiainen, M., Lang, M., Mõttus, M., Kuusk, A., Nilson, T., Kuusk, J. \& Lükk, T., 2008. Multiangular reflectance properties of a hemiboreal forest: An analysis using CHRIS PROBA data. Remote Sensing of Environment,112 (5), pp.2627-2642 
651

652

653

654

655

656

657

658

659

660

661

662

663

664

665

666

667

668

669

670

671

Reynolds, M., Foules, M.J., Slafer, G.A., Berry, P., Parry, M.A., Snape, J.W., Angus, W.J. (2009).

Raising wheat potential. Journal of Experimental Botany. 60: pp. 1899-1918.

Roggo, Y., Duponchel, L., Huvenne, J.P. (2003). Comparison of supervised pattern recognition methods with McNemar's statistical test: application to qualitative analysis of sugar beet by near-infrared spectroscopy. Analytica Chimica Acta, 477(2): pp. 187-200.

Rouse, J.W. Jr., R.H. Haas, D.W. Deering, J.A. Schell and J.C. Harlan. 1974. Monitoring the Vernal Advancement and Retrogradation (Green Wave Effect) of Natural Vegetation. NASA/GSFC Type III Final Report, Greenbelt, MD., 371.

Sankaran, S., Mishra, A., Ehsani, R., \& Davis, C. (2010). A review of advanced techniques for detecting plant diseases. Computers and Electronics in Agriculture, 72(1), 1-13.

Schmale, D. G., III, \& Bergstrom, G. C. (2003). Fusarium head blightin wheat. The Plant Health Instructor. http://dx.doi.org/10.1094/PHI-I-2003-0612-01.

Smith, S. W. (1999). The Scientist and Engineer's Guide to Digital Signal Processing. 2nd ed. San Diego: California Technical Publishing, (Chapter 2 pp 13-16).

Sohne, W. (1958). Fundamentals of pressure distribution and soil compaction under tractor tires. Agricultural Engineering, 39, 290

Tekin, Y., Kuang, B., \& Mouazen, A. M. (2013). Potential of on-line visible and near infrared spectroscopy for measurement of $\mathrm{pH}$ for deriving variable rate lime recommendations. Sensors, 13(8), 10177-10190.).

Van Beek, J., Tits, L., Somers, B., \& Coppin, P. (2013). Stem water potential monitoring in pear orchards through WorldView-2 multispectral imagery. Remote Sensing, 5(12), 6647-6666. 
672

673

674

675

676

677

678

679

680

681

682

683

684

685

686

687

688

689

690

Van der Meer, F. and De Jong, S.M., 2000. Improving the results of spectral unmixing of Landsat Thematic Mapper imagery by enhancing the orthogonality of endmembers. International Journal of Remote Sensing,21(15): pp. 2781-2797.

Webster, R. (2007). Analysis of variance, inference, multiple comparisons and sampling effects in soil research. European Journal of Soil Science, 58(1), pp. 74-82.

West, J.S., Bravo, C., Oberti, R., Lemaire, D., Moshou, D. and McCartney, H.A., 2003. The potential of optical canopy measurement for targeted control of field crop diseases. Annual review of Phytopathology, 41(1), pp. 593-614.

Wu, D., Feng, L., Zhang, C., \& He, Y., (2008). Early detection of Botrytis cinerea on eggplant leaves based on visible and near-infrared spectroscopy. Transactions of the ASABE 51 (3), pp. 1133-1139.

Yuan, L., Pu, R., Zhang, J., Wang, J. and Yang, H. (2016). Using high spatial resolution satellite imagery for mapping powdery mildew at a regional scale. Precision Agriculture, 17(3): pp. $332-348$.

Zadoks, J.C.; T.T. Chang, C.F. Konzak (1974). "A decimal code for the growth stages of cereals.". Weed Research 14 (6): 415-421. doi:10.1111/j.1365-3180.1974.tb01084.x.

Zhong, C., Ding, Y., \& Fu, J. (2011). Image Forward Motion Compensation Method for Some Aerial Reconnaissance Camera Based on Neural Network Predictive Control. Advanced Research on Computer Science and Information Engineering. 152 (1), pp. 237-244. 